\title{
OPEN Intermittent decoherence blockade in a chiral ring environment
}

\begin{abstract}
Salvatore Lorenzo ${ }^{1}$, Stefano Longhi ${ }^{2}$, Albert Cabot $^{3}$, Roberta Zambrini ${ }^{3}$ \& Gian Luca Giorgi ${ }^{3 凶}$
It has long been recognized that emission of radiation from atoms is not an intrinsic property of individual atoms themselves, but it is largely affected by the characteristics of the photonic environment and by the collective interaction among the atoms. A general belief is that preventing full decay and/or decoherence requires the existence of dark states, i.e., dressed light-atom states that do not decay despite the dissipative environment. Here, we show that, contrary to such a common wisdom, decoherence suppression can be intermittently achieved on a limited time scale, without the need for any dark state, when the atom is coupled to a chiral ring environment, leading to a highly non-exponential staircase decay. This effect, that we refer to as intermittent decoherence blockade, arises from periodic destructive interference between light emitted in the present and light emitted in the past, i.e., from delayed coherent quantum feedback.
\end{abstract}

Spontaneous emission is a fundamental process in quantum optics and quantum electrodynamics ${ }^{1-3}$. While in the most typical cases it is described by an exponential decay of a quantum (atomic or solid state) system towards its ground state, accompanied by an irreversible emission of a photon ${ }^{4}$, the properties of the surrounding photonic environment $^{5,7}$, as well as measurement ${ }^{8}$, or collective effects ${ }^{9,10}$, can largely affect spontaneous emission, with consequences ranging form control of single-photon sources to decoherence.

Dimension and geometric constraints of the photonic environment (like cavities ${ }^{5}$ ), continuous or discretemode structures of the reservoir ${ }^{11}$, as well as engineered surrounding media (for instance exhibiting band-gaps ${ }^{7}$ ), can significantly enhance or inhibit the decay rate of a single emitter. Recently, more complex photonic environments have been shown to be powerful resources for controlling light-emitter interaction in unprecedented ways $^{12-15}$.

Coupling one or more atoms to one-dimensional chiral waveguides or topological photonic structures, that break time reversal symmetry, enables to control the directionality of spontaneous emission and to deeply modify photon-mediated interactions, with major applications in the design of integrated non-reciprocal single-photon devices, spin-photon interfaces, and in the synthesis of novel quantum states such as entangled spin states and photonic clusters states ${ }^{16-23}$. Likewise, 'giant' artificial atoms, in which the atomic dimension greatly exceeds the 'photon' wavelength and the time spent by light to cross the atom can not be neglected, provide a new paradigm of atom-field interaction ${ }^{24-33}$. Since the atom cannot be considered point-like anymore, spontaneous emission ceases to be exponential and the decay dynamics is described by a differential-delayed equation ${ }^{25,27,30,33}$, displaying strictly non-Markovian (memory) effects arising from delayed coherent quantum feedback ${ }^{34-36}$. Similar memorylike effects are also found in ordinary (point-like) atoms in the presence of mirrors or retardation effects ${ }^{37-46}$.

One among the most striking phenomena achieved through complex environment engineering is the possibility to inhibit spontaneous emission and dechoerence under certain geometric conditions, i.e. the stabilization of quantum superposition states in the presence of dissipation or other forms of decay channels or dephasing. This goal is of major relevance in different contexts ranging from quantum computation, where limiting effects of decoherence ${ }^{47}$ and decoherence-free-space have been broadly studied ${ }^{48,49}$, to quantum biology ${ }^{50-52}$ and quantum chemistry $^{53,54}$, where pure dephasing and non-radiative channels are the main sources that destroy electronic coherence in molecular dynamics. Such a decoherence/decay blockade stems from the appearance of dressed light-matter states, commonly known as dark states, or else bound states in the continuum, that do not decay despite the dissipative environment. The existence of dark states and their ability to prevent quantum decay via destructive interference among different decay channels has been known since long time and studied in several areas of physics ${ }^{55-74}$, along with the related concept of decoherence-free subspaces ${ }^{75}$, i.e. regions in Hilbert space which are not affected by decoherence. A fully open question is whether spontaneous emission and decoherence

${ }^{1}$ Dipartimento di Fisica e Chimica, Universitá degli Studi di Palermo, Via Archirafi 36, 90123 Palermo, Italy. ${ }^{2}$ Dipartimento di Fisica, Politecnico di Milano, Piazza L. da Vinci 32, 20133 Milan, Italy. ${ }^{3}$ IFISC (UIB-CSIC), Instituto de Fisica Interdisciplinar y Sistemas Complejos, 07122 Palma de Mallorca, Spain. ${ }^{\square}$ email: gianluca@ ifisc.uib-csic.es 
(a)

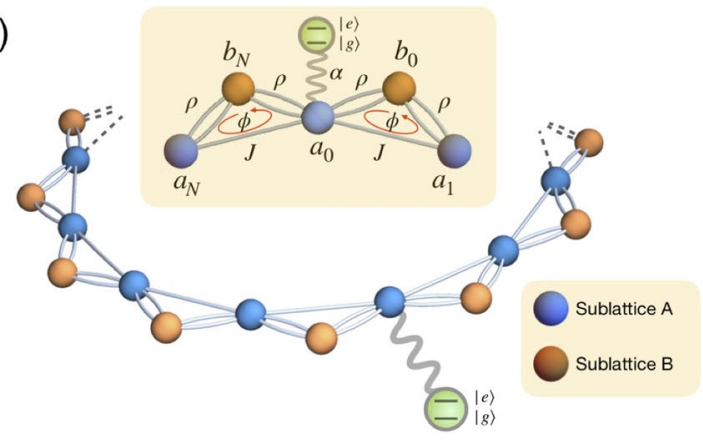

(b)

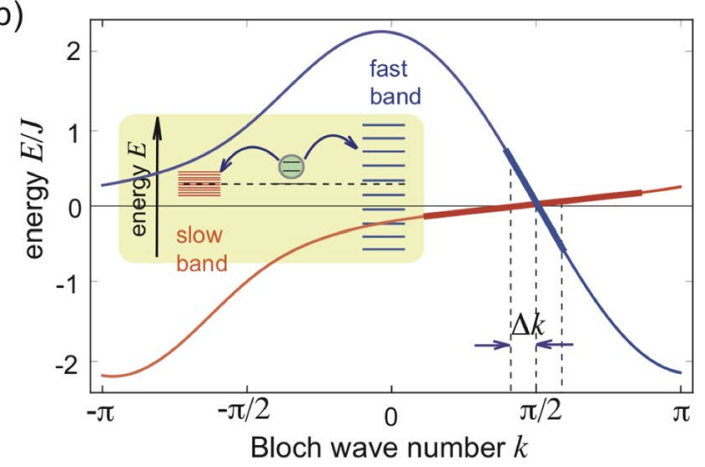

Figure 1. (color online) (a) Schematic of a two-level quantum emitter decaying on a chiral sawtooth photonic lattice. The lattice comprises two sublattices A and B, with hopping constants $J$ and $\rho$ (represented by single and double lines), on a ring geometry. A synthetic magnetic flux $\phi$ is applied in each plaquette of the lattice (see the inset). (b) Energy diagram (dispersion curves) of the bipartite ring lattice for $\rho / J=0.5$ and $\phi=\pi / 2$. Note the band closing point at $k=\pi / 2$ due to the $\phi=\pi / 2$ flux condition, and the existence of fast and slow bands crossing at the gap closing point, corresponding to counter-propagating modes with fast and slow group velocities $\Omega^{ \pm}$. Quantization of the Bloch wave number $\Delta k=2 \pi / N$ due to the ring boundary conditions introduces two energy scales (i.e. energy quantization) for fast and slow bands, into which the quantum emitter decays (see the inset).

can be inhibited, at least transiently or intermittently, in the absence of any decoherence-free subspace, or even though the atom-light system does not show any dark state.

In this work we show rather surprisingly that, harnessing the idea of delayed coherent quantum feedback in a reservoir with effective discrete and continuous mode structure, a point-like atom emitting in a chiral ring photonic waveguide, sustaining slow and fast counter-propagating photonic modes, undergoes intermittent decoherence suppression on a fast time scale, displaying an exotic staircase decay dynamics. Such an effect, that we refer to as intermittent decoherence blockade, arises from periodic destructive interference between light emitted in the present, both in fast and slow photonic modes, and light emitted in the past in the fast photonic modes. Due to the different group velocities of counter-propagating chiral modes in the ring, two different time scales are involved in the decay dynamics, which are determined by the energy level spacing in the slow and fast bands of the ring waveguide. On the fast time scale, which is of major interest in our work, the atom turns out to be effectively coupled with both a continuous set of modes (the modes of the slow ring band) into which irreversible decay and decoherence occur, and a discrete set of modes (the modes of the fast ring band), which provide delayed feedback and re-coherence in the atom and are thus ultimately responsible for the intermittent decoherence blockade predicted in our work. Clearly, on the long time scale, determined by the energy spacing between the levels in the band with the slow group velocity, the full discrete nature of the reservoir will lead to a fully coherent and unitary dynamics, and the system will almost surely return to its initial state according to the quantum recurrence theorem ${ }^{76}$.

\section{Results}

Decoherence dynamics of an atom coupled to a chiral ring. We consider the decay/decoherence dynamics of a two-level atom coupled to the radiation modes of an engineered chiral bath with broken time reversal symmetry. The photonic bath realizes a chiral sawtooth waveguide ${ }^{21,22}$, consisting of a bipartite lattice of cavities/resonators composed by two sublattices A and B in a ring geometry, and threaded by a synthetic gauge field $\phi$ in each plaquette, as schematically depicted in Fig. 1a. Such a model system has been investigated in some recent works and can be physically implemented in different platforms, such as squids, cold atoms, and integrated photonic circuits ${ }^{21-23}$. The bath is governed by the nearest-neighbor tight-binding Hamiltonian ${ }^{22}$

$$
\hat{H}_{B}=\sum_{n=1}^{N}\left\{\omega\left(\hat{a}_{n}^{\dagger} \hat{a}_{n}+\hat{b}_{n}^{\dagger} \hat{b}_{n}\right)+\left[\hat{a}_{n}\left(J \hat{a}_{n+1}^{\dagger}+\rho e^{-i \phi} \hat{b}_{n-1}^{\dagger}+\rho \hat{b}_{n}^{\dagger}\right)+\text { h.c. }\right]\right\},
$$

where $\hat{a}_{n}$ and $\hat{b}_{n}$ are the annihilation operators of the n-th $a$ and $b$ modes with same frequency $\omega$ (henceforth we will work in a frame rotating at $\omega$ ). The constants $J$ and $\rho$ are the nearest-neighbour coupling between the A lattice sites and the hopping strength between the $a$ and $b$ modes respectively, as shown in Fig. 1a. This simple one-dimensional model admits complex couplings between lattice vertices, defining an effective magnetic flux per loop denoted by $\phi$ in Eq. (1). As detailed in Ref. ${ }^{22}$, the saw-tooth lattice can be realized using superconducting qubits by coupling several single-loop plaquettes, experimentally implemented in Ref. ${ }^{77}$. In such scenario, typical lifetimes for the tight-binding Hamiltonian modes can be extracted from the experimental measurements of Refs. ${ }^{77,78}$ and range from a few $\mu$ s to a few tens of $\mu$ s. Given the bipartite nature of the bath, the energy spectrum of $\hat{H}_{B}$ comprises two energy bands (Fig. 1b), with dispersion relations given by (see Methods) 


$$
E_{k}^{ \pm}=J \cos k \pm \sqrt{J^{2} \cos ^{2} k+2 \rho^{2}(1+\cos (\phi+k))} .
$$

A non-vanishing magnetic flux $\phi$ breaks the time reversal symmetry of $\hat{H}_{B}$, i.e. $E_{-k}^{ \pm} \neq E_{k}^{ \pm}$. Remarkably, for $\phi=\pi / 2$ the intraband gap closes, giving raise to a band crossing at $k=\pi / 2^{22}$ with energy $E_{\pi / 2}^{ \pm}=0$. Near the band crossing point, the dispersion relations of the two bands show an almost linear behavior, resulting in a slow $\left(\Omega^{+}\right)$and fast $\left(\Omega^{-}\right)$group velocities with opposite signs for the two bands, see Fig. $1 \mathrm{~b}$.

A point-like emitter decays into the chiral bath via a weak coupling to the radiation mode of one resonator of sublattice A. Modelling the emitter as a two level system, $\{|g\rangle,|e\rangle\}$, with energy separation $\omega_{e}$, we write the free Hamiltonian of the emitter and interaction Hamiltonian with the bath as

$$
\hat{H}_{e}+\hat{H}_{i n t}=\omega_{e}|e\rangle\langle e|+\alpha\left(\hat{\sigma}^{-} \hat{a}_{0}^{\dagger}+\hat{\sigma}^{+} \hat{a}_{0}\right),
$$

where $\sigma^{ \pm}$are the usual ladder Pauli operators. As shown in the Methods, the bath Hamiltonian can be diagonalized introducing slow and fast modes $s_{k}$ and $f_{k}$,

$$
\hat{H}_{B}=\sum_{k} E_{k}^{-} \hat{s}_{k}^{\dagger} \hat{s}_{k}+E_{k}^{+} \hat{f}_{k}^{\dagger} \hat{f}_{k} .
$$

In this representation the interaction part of Eq. (3) results to be

$$
H_{\text {int }}=\sum_{k} \frac{\alpha_{k}^{-}}{\sqrt{N}} \hat{\sigma}^{+} \hat{s}_{k}+\frac{\alpha_{k}^{+}}{\sqrt{N}} \hat{\sigma}^{+} \hat{f}_{k}+\text { h.c. }
$$

with couplings $\alpha_{k}^{ \pm}=\alpha E_{k}^{ \pm} / N_{k}^{ \pm}$, where $N_{k}^{ \pm}=\sqrt{\left(E_{k}^{ \pm}\right)^{2}-E_{k}^{+} E_{k}^{-}}$.

Supposing that the initial state is $|\phi(0)\rangle=|e\rangle \otimes|v a c\rangle(|v a c\rangle$ denotes the vacuum state of the bath), it will evolve into $|\phi(t)\rangle=\varepsilon(t)|e\rangle \otimes|v a c\rangle+\sum_{k, \pm} c_{k}^{ \pm}(t)|g\rangle \otimes\left|\psi_{k}^{ \pm}\right\rangle$, with $\varepsilon(0)=1$. Following standard procedures (see the Methods), we arrive at the integral differential equation for the emitter excitation amplitude

$$
\dot{\varepsilon}(t)=-i \omega_{e} \varepsilon(t)-\frac{1}{N} \int_{0}^{t} d s \varepsilon(t-s) \sum_{k, \pm}\left|\alpha_{k}^{ \pm}\right|^{2} e^{-i E_{k}^{ \pm} s} .
$$

For $\phi=\pi / 2$ the two dispersion curves (2) can be linearized near $k=\pi / 2$. Assuming the emitter resonant with modes near the crossing point $\left(\omega_{e}=0\right.$, or $\omega_{e}=\omega$ in the lab frame) the equation for excitation amplitude (6) becomes

$$
\dot{\varepsilon}(t) \simeq-\frac{1}{N} \int_{0}^{t} d s \varepsilon(t-s) \sum_{k, \pm}\left|\alpha_{\pi / 2}^{ \pm}\right|^{2} e^{-i \Omega^{ \pm}\left(k-\frac{\pi}{2}\right) s},
$$

where $\Omega^{ \pm}=-J \pm \sqrt{J^{2}+\rho^{2}}$. Note that, according to Eqs. (6) and (7), the amplitude $\epsilon(t)$ and its derivative $\dot{\epsilon}(t)$ are continuous functions of time $t$, as it should be on physical grounds, However, as discussed in the next section, for a large number $N$ of sites in the ring the numerically-computed evolution of $\epsilon(t)$ displays rather sharp changes at time instants corresponding to periodic feedback from the ring. Such a scenario, which is similar to the dynamical behavior observed for a point-like two-level atom radiating in front of a mirror ${ }^{37,39,40}$, can be captured by approximating the exact integro-differential equations $(6,7)$ with a differential-delayed equation ${ }^{32,40}$. To this aim, let us note that in the large $N$ limit the sum over $k$ on the right hand side of Eq. (7) can be approximated by Dirac comb

$$
\sum_{k} e^{-i \Omega^{ \pm} k s} \sim e^{-i \frac{N-1}{N} \pi \Omega^{ \pm} s} \frac{N}{\left|\Omega^{ \pm}\right|} \sum_{n=0}^{\infty}(-1)^{n(N+1)} \delta\left(s-\frac{n N}{\left|\Omega^{ \pm}\right|}\right) .
$$

In this way the time integration in (7) can be performed leading to

$$
\dot{\varepsilon}(t)=-\frac{1}{2} \gamma_{0} \varepsilon(t)-\sum_{n=1}^{\infty} \sum_{ \pm} \gamma_{n}^{ \pm} \varepsilon\left(t-n T^{ \pm}\right) \Theta\left(t-n T^{ \pm}\right),
$$

where

$$
\gamma_{n}^{ \pm}=\frac{\left|\alpha_{\pi / 2}^{ \pm}\right|^{2}}{\left|\Omega^{ \pm}\right|} e^{ \pm i \pi n \frac{N}{2}}, \quad T^{ \pm}=\frac{N}{\left|\Omega^{ \pm}\right|},
$$

$\gamma_{0}=\gamma_{0}^{+}+\gamma_{0}^{-}$, and $\Theta(x)$ stands for the Heaviside step function. From inspection of Eq. (9) we anticipate that, unless $N=2(2 M)$, the damping rates exhibit a peculiar alternate change of sign at any round, increasing $n$. Note that by solving Eq. (8), the behavior of coherences is also known. In fact, the reduced density matrix of the two-level atom at time $t$ is given by

$$
\rho(t)=\left(\begin{array}{cc}
|\varepsilon(t)|^{2} \rho_{e e} & \varepsilon(t) \rho_{e g} \\
\varepsilon(t)^{*} \rho_{g e} & \left(1-|\varepsilon(t)|^{2}\right) \rho_{e e}+\rho_{g g}
\end{array}\right),
$$


where $\rho_{j k}=\langle j|\rho(0)| k\rangle$ with $j, k=g, e$ are the entries of the possible mixed initial atomic density matrix $\rho(0)$. The first term in the right hand side of (8) represent the initial decay into "both" channels, while the successive terms take into account back excitation from the bath into the emitter when the slow and fast modes make entire loops through the ring, i.e. in the presence of delayed coherent quantum feedback. The decay dynamics is thus governed by three different time scales: (i) the decay time $T_{d}=1 / \gamma_{0}$, i.e. the inverse of the decay rate $\gamma_{0}=\gamma_{0}^{+}+\gamma_{0}^{-}$as determined by the usual Fermi golden rule in the weak-coupling and $N \rightarrow \infty$ limits or, equivalently, by the spectral density, that is, by the Fourier transform of the sum in Eq. (6), in a master equation approach; this term is proportional to $\alpha^{-2}$; (ii) and (iii) the feedback delay times $T^{ \pm}=N /\left|\Omega^{ \pm}\right|$for the fast and slow decay channels, depending only on bath parameters. Note that, in the limit $\rho / J \rightarrow 0$, the slow decay channel corresponds to a vanishing group velocity $\Omega^{+} \rightarrow 0$ (flat band limit), i.e. in an extremely long delay feedback time $T^{+}$. Here we restrict our analysis considering the decay dynamics on a time scale shorter than $T^{+}$, so that the slow decay channel (slow band) can be regarded as a true bath with continuous energy spectrum, into which the point emitter continuously decays. For times longer than $\sim T^{-}$, the discreteness of energy levels in the fast band cannot be neglected. So, on the one hand, the atom decays into a very continuum of modes (slow band), while, on the other hand, it is periodically fed by the discrete modes of the fast band, which are responsible for delayed coherent quantum feedback. For a detailed discussion about delayed quantum feedback induced by reservoirs with continuous and discrete modes see Ref. ${ }^{11}$.

Intermittent decoherence blockade in a chiral bath: delayed coherent quantum feedback and staircase dynamics. According to our theoretical analysis, two very distinct dynamical scenario are expected depending on whether $T_{d} \ll T^{-}$or $T_{d} \gg T^{-}$. While in the former case the decay dynamics shows typical multiple revivals, as observed e.g. in giant atom decay dynamics ${ }^{27}$, in the latter case a fully distinct behavior is observed, characterized by intermittent decays separated by intervals of decay suppression, corresponding to intermittent decoherence blockade. We computed the exact decay dynamics of $\epsilon(t)$ by numerically solving the coupled equations (15) given in the Methods, which are equivalent to the integro-differential equation (6).

An example of multiple revivals in the decoherence dynamics is shown in Fig. 2a, where the numericallycomputed solution of $(6)$ is reported for $T_{d} \ll T^{-}$and $T^{+}=2 \sqrt{2}+3$. Note that, after each time interval of duration $T^{-}$, excitation almost completely decays into the bath, however incomplete recoherence is periodically observed due quantum feedback from the fast channel into the atom. On other hand when $T_{d} \gg T^{-}$, i.e. $\alpha \ll 1$, a surprising result is observed provided that $N=2(2 s+1)$ with $s$ integer, namely decoherence can be intermittently suppressed (see the Methods for the dynamics with different values of $N$ ). An example of the decoherence dynamics in this regime obtained by simulation of the exact dynamics, Eq. (6) is shown in Figs. 2b,c and 3a. Clearly, the decay of coherence $|\epsilon(t)|$ largely deviates from an exponential decay during time $T_{d}$ and, most importantly, shows a nearly staircase behavior, where decoherence is inhibited at alternating time intervals of duration $T^{-}$, while it displays an almost linear decay outside these intervals. In the inset of Fig. $2 \mathrm{~b}$, we show that this peculiar behavior can be found looking at basis-independent indicators, such as the purity (defined as the trace of $\left.\rho^{2}(t)\right)$ and the von Neumann entropy $S(\rho(t))=-\operatorname{Tr}[\rho(t) \log \rho(t)]$. Figure 2c compares the intermittent decoherence blockade dynamics, arising from delayed quantum feedback of the ring bath geometry, with the conventional nearly-exponential decay dynamics that one would observe in the continuous limit $N=\infty$, where the feedback is absent. For times $t<T^{-}$, the decay dynamics in the two cases is clearly the same. In particular, in the very early stage near $t=0$, the decay is parabolic (Zeno interval, panel A), as it should be. Likewise, in the early stage the purity dynamics shows a parabolic (Gaussian) behavior, which is not however visible on the time scale depicted in the inset of Fig. 2b. In the continuous limit $N=\infty$, after the Zeno time the decay becomes exponential with a decay rate that turns out to be in excellent agreement with the Fermi golden rule prediction $\gamma_{0}$. Note that, since $\gamma_{0} T^{-} \ll 1$, the exponential decay in the range $\left(0, T^{-}\right)$is very close to a linear decay. The dynamical behavior near the time $t=T^{-}$is shown in panel $\mathrm{B}$. As one can see, while in the continuous limit the decay is not interrupted (absence of delayed feedback from the bath), destructive interference leading to almost stopping of the decay is observed for finite $N$.

In order to better understand this intermittent decoherence blockade, one should look at the solution to (8), which can be given in terms of confluent hypergeometric functions, as shown in the Methods. In particular, the Taylor expansion of $\varepsilon(t)$ up to the second order in $\alpha$, as obtained from the solution to Eq. (8), reads explicitly

$$
\varepsilon(t) \sim\left\{\begin{array}{l}
1-\frac{1}{2} \gamma_{0}\left(t-n T^{-}\right) \quad \text { for } \quad(2 n) T^{-} \leq t \leq(2 n+1) T^{-} \\
1-\frac{1}{2} \gamma_{0}(n+1) T^{-} \text {for } \quad(2 n+1) T^{-} \leq t \leq(2 n+2) T^{-},
\end{array}\right.
$$

with $n=\{0,1,2,3, \ldots\}$ such that $n T^{-}<T^{+}$. It is evident now, how the coherence decay results intermittently suppressed, for an interval of time equal to $T^{-}$. The intermittent decoherence blockade can be traced back to destructive interference between light emitted in the past in the fast band and light emitted in the present in both fast and slow bands of the bath, as shown in Fig. 3. The figure depicts the total excitation present in sublattices $A$ and $B$. In a counter intuitive way, we see that every time the "two waves", slow and fast, meet [this happen at times $t^{*}=n T^{-} T^{+} /\left(T^{-}+T^{+}\right)$, vertical solid lines in Fig. 3], the excitation accumulated on sublattice $A$ is transferred to sublattice $B$, while the overall decay of the emitter is blocked at different alternating intervals (vertical dotted lines in Fig. 3). We emphasize that such an intermittent suppression of decoherence arising from delayed coherent quantum feedback does not require the existence of dark states, contrary to other decoherence suppression methods based on delayed feedback ${ }^{34,44,45}$. Indeed, as shown in the Methods, the full Hamiltonian $\hat{H}=\hat{H}_{B}+\hat{H}_{e}+\hat{H}_{\text {int }}$ does not sustain eigenmodes localized near the emitter.

Finally, it should be mentioned that a necessary condition for the observation of the intermittent (staircase) decoherence behavior is the resonance between the frequency of the emitter and the band crossing energy of the bath, which allowed us to perform the linearization of Eq. (7). This resonance would be lost if for instance we 
(a)

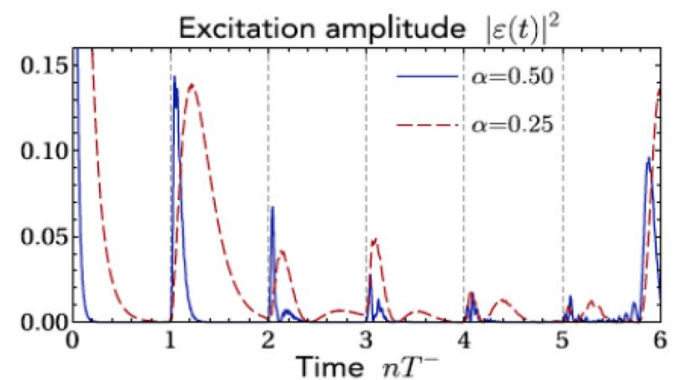

(c)

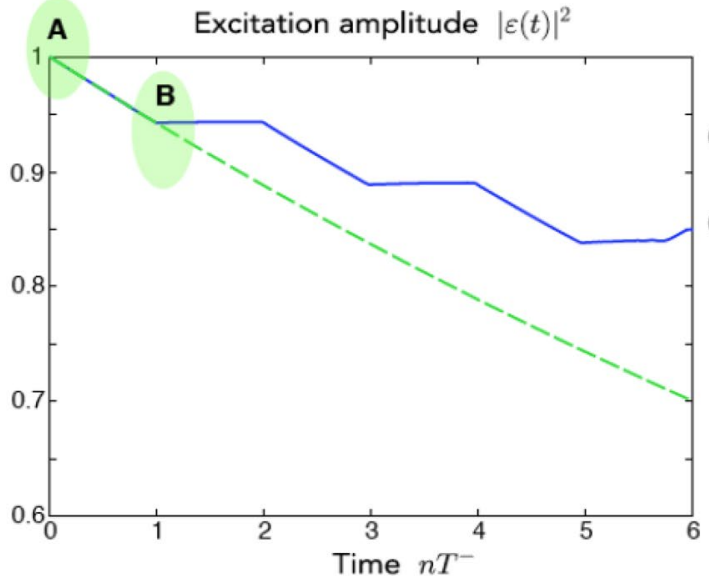

(b)
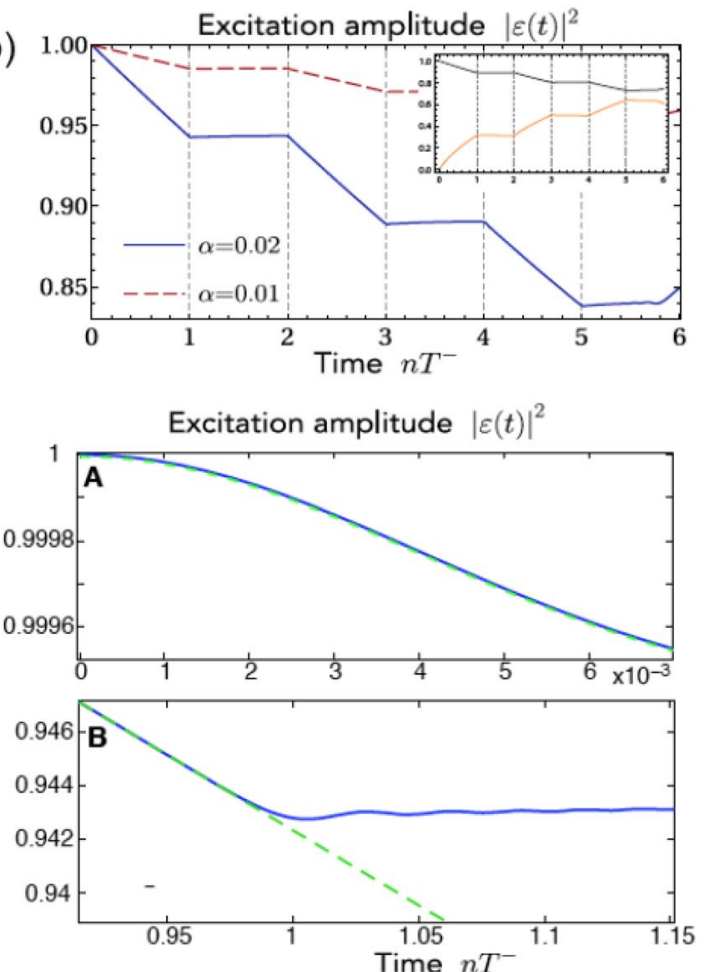

Figure 2. (color online) (a,b) Evolution of the excitation amplitude $|\varepsilon(t)|^{2}$, solution of Eq. (6) for $N=502$, $J=\rho=1$ and different values of $\alpha$. (a) $(\alpha=0.25$ and $\alpha=0.50)$ In this regime a non exponential decay dynamics is observed, in which multiple revivals occur for $t=n T^{-}$. (b) $(\alpha=0.01$ and $\alpha=0.02)$ In this regime intermittent blockade of the decay is observed until the time $t \sim 6 T^{-}$; at longer times feedback from the slow modes arises, which breaks intermittent decay suppression. Inset: evolution of purity (black) and von Neumann entropy (orange) for $\alpha=0.02$. (c) Comparison between decay dynamics for $\alpha=0.02, N=502$ [solid curve, as in panel (b)] and for $\alpha=0.02, N=\infty$ (dashed curve, continuous limit without delayed feedback). Note that for $0<t<T^{-}$the two curves are overlapped. The plots on the right side in A and B depict an enlargement of the decay dynamics near the early time $t=0$, where the decay is parabolic (Zeno dynamics, panel A), and near the time $t=T^{-}$, where stopping of decoherence decay is observed in the finite $N$ case due to coherent delayed feedback.

\section{(a) Excitation amplitudes}

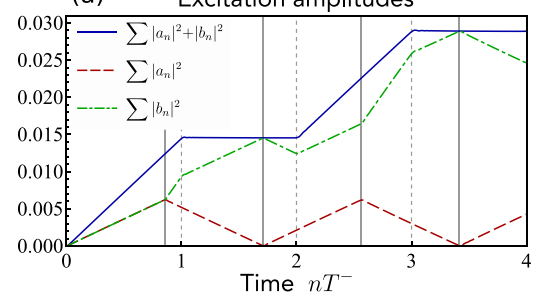

(b)

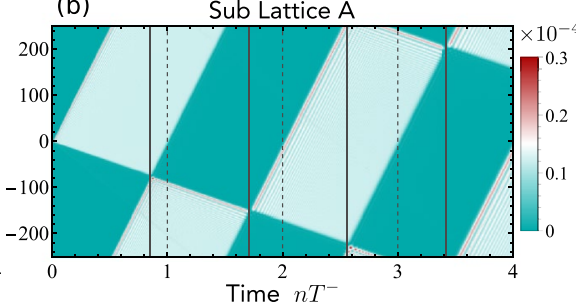

(c)

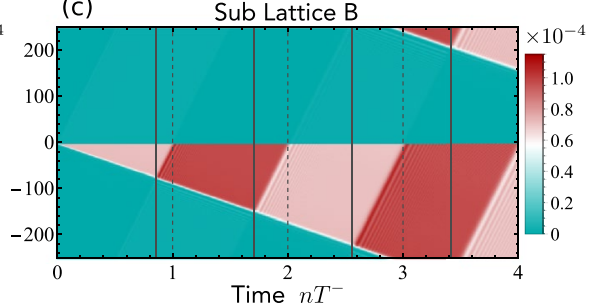

Figure 3. (color online) (a) Temporal evolution of the total excitation amplitude relative to sublattices A and B for in the intermittent decay blockade regime $(N=502, J=\rho=1$ and $\alpha=0.01)$. (b),(c) Corresponding detailed evolution of the excitation amplitudes in sublattices A and B on a pseudocolor map (see the "Methods" for the definition of such amplitudes). In each plot, vertical lines corresponds to times (solid) at which slow and fast waves meet and to times (dotted) at which the fast one make a complete loop.

changed the interaction Hamiltonian, defined by Eq. (3), by considering a pure dephasing coupling of the form $\hat{H}_{\text {dep }}=\alpha \hat{\sigma}_{z}\left(\hat{a}_{0}+\hat{a}_{0}^{\dagger}\right)$. In this case, the decoherence caused by the bath (only observable if the non-diagonal elements of the emitter density matrix were populated at time $t=0$ ) would be proportional to the scalar product $\left\langle v a c\left|e^{-i\left(H_{B}+H_{\text {dep }}^{\prime}\right) t}\right| v a c\right\rangle$ (where $\hat{H}_{\text {dep }}^{\prime}=\alpha\left(\hat{a}_{0}+\hat{a}_{0}^{\dagger}\right)$ is obtained from replacing $\hat{\sigma}_{z}$ with its eigenvalue 1 over the excited state of the emitter in $\hat{H}_{\text {dep }}$ ) which would depend on the whole spectrum and would not display any sign of resonance. 


\section{Discussion}

Quantum emitters coupled to photonic waveguides represent a powerful integrated platform for complex quantum networks. While in typical scenarios the interaction between atoms and light can be treated using a master equation obeying the Born-Markov approximation, nontrivial phenomena can emerge beyond this limit. In particular, the Markovian approximation clearly fails in the presence of delayed coherent quantum feedback, where one has to take into account the effects of the finite propagation speed of light, which introduces an effective memory.

The possibility to block decoherence is generally associated with the presence of frequency gap environments or, through a less trivial mechanism, due to the presence of bound states into the continuum. In this work we showed a different scenario that exploits the chirality of the environment, which is responsible, at the same time, for the decay into a continuum of modes and delayed feedback due to a discrete set of modes. Indeed, the interference effects of delayed coherent quantum feedback enables intermittent decoherence suppression. Our results go beyond previous findings, which linked the presence of coherent quantum feedback to the emergence of dressed light-atom dark states that cause light to be trapped around the emitter, as for instance in the famous atom-in-front-of-a-mirror example. We showed that chiral waveguides can be exploited to generate nontrivial interference patterns, as they are able to separate fast and slow wavepackets, altering in this way the effect of delayed feedback. The surprising result is the total suppression of decoherence during finite time windows (whose length can be tailored modifying the system parameters) even in the absence of dark states. As an additional comment, we point out that, since the photonic environment can be readily reconfigured by tuning the gauge phase $\phi$, we can open a wide gap and fully stop the decay of the quantum emitter on demand. Moreover, since the intermittent decoherence blockade is ultimately an interference effect which is very sensitive to the gauge phase $\phi$, besides controlling decoherence our setup could be of relevance for quantum sensing. To summarize, our results suggest that chiral waveguides together with delayed coherent quantum feedback represent a powerful reservoir engineering tool of potential relevance in quantum manipulation and control, quantum sensing and quantum networks.

\section{Methods}

$\hat{H}_{B}$ diagonalization. Under periodic boundary conditions, we can introduce momentum operators, $a_{k}\left(\hat{b}_{k}\right)=1 / \sqrt{N} \sum_{n=1}^{N} e^{-i k n} \hat{a}_{n}\left(\hat{b}_{n}\right)$, that allow us, moving to the rotating frame at the bare energy $\omega$, to rewrite the Hamiltonian $\hat{H}_{B}$ in the following form

$$
\hat{H}_{B}=\sum_{k}\left(\hat{a}_{k}^{\dagger} \hat{b}_{k}^{\dagger}\right) h_{k}\left(\begin{array}{l}
\hat{a}_{k} \\
\hat{b}_{k}
\end{array}\right),
$$

where

$$
h_{k}=\left(\begin{array}{cc}
J\left(e^{-i k}+e^{i k}\right) & g_{k}^{*} \\
g_{k} & 0
\end{array}\right),
$$

with $g_{k}=\rho\left(1+e^{-i(\phi+k)}\right)$. Each $h_{k}$ can be diagonalized with its respective unitary matrix

$$
U_{k}=\left(\begin{array}{cc}
E_{k}^{-} / N_{k}^{-} & E_{k}^{+} / N_{k}^{+} \\
g_{k} / N_{k}^{-} & g_{k} / N_{k}^{+}
\end{array}\right),
$$

with $N_{k}^{ \pm}=\sqrt{\left(E_{k}^{ \pm}\right)^{2}+\left|g_{k}\right|^{2}}$. In this way, the slow and fast modes $s_{k}$ and $f_{k}$ are defined as

$$
\left(\begin{array}{l}
\hat{s}_{k} \\
\hat{f}_{k}
\end{array}\right)=U\left(\begin{array}{l}
\hat{a}_{k} \\
\hat{b}_{k}
\end{array}\right)=\left(\begin{array}{l}
\frac{E_{k}^{-}}{N_{k}^{-}} \hat{a}_{k}+\frac{E_{k}^{+}}{N_{k}^{+}} \hat{b}_{k} \\
\frac{g_{k}}{N_{k}^{-}} \hat{a}_{k}+\frac{g_{k}}{N_{k}^{+}} \hat{b}_{k}
\end{array}\right) .
$$

Derivation of equation for emitter excitation amplitude. An initial state of the form $|\phi(0)\rangle=|e\rangle \otimes \mid$ vac $\rangle$ will evolve in time as

$$
|\phi(t)\rangle=\varepsilon(t)|e\rangle \otimes|v a c\rangle+\sum_{k, \pm} c_{k}^{ \pm}(t)|g\rangle \otimes\left|\psi_{k}^{ \pm}\right\rangle,
$$

where $\varepsilon(0)=1, c_{k}^{ \pm}(0)=0$, and where $\left|\psi_{k}^{+}\right\rangle=\hat{s}_{k}^{\dagger}|v a c\rangle,\left|\psi_{k}^{-}\right\rangle=\hat{f}_{k}^{\dagger}|v a c\rangle$. The time-dependent Schrödinger equation yields the set of coupled differential equations

$$
\begin{aligned}
i \dot{\varepsilon}(t) & =\omega_{e} \varepsilon(t)+\frac{1}{\sqrt{N}} \sum_{k \pm} \alpha_{k}^{ \pm} c_{k}^{ \pm}(t), \\
i \dot{c}_{k}^{ \pm}(t) & =E_{k}^{ \pm} c_{k}^{ \pm}(t)+\frac{1}{\sqrt{N}} \alpha_{k}^{ \pm} \varepsilon(t) .
\end{aligned}
$$

Formally integrating the latter and substituting in the former, one arrive at the integral differential equation for the emitter excitation amplitude $\varepsilon(t)$ : 


$$
\dot{\varepsilon}(t)=-i \omega_{e} \varepsilon(t)-\frac{1}{N} \int_{0}^{t} d s \sum_{k \pm}\left|\alpha_{k}^{ \pm}\right|^{2} \varepsilon(s) e^{-i E_{k}^{ \pm}(t-s)} .
$$

Absence of dark states. In the single-excitation sector, the energy spectrum $E$ and corresponding eigenstates of the full Hamiltonian $\hat{H}=\hat{H}_{B}+\hat{H}_{e}+\hat{H}_{\text {int }}$ are obtained by solving the linear eigenvalue problem

$$
\begin{aligned}
E a_{n}= & J\left(a_{n+1}+a_{n-1}\right)+\rho b_{n}+\rho \exp (-i \phi) b_{n-1} \\
& +\alpha \epsilon \delta_{n, 1} \\
E b_{n}= & \rho a_{n}+\rho \exp (i \phi) a_{n-1} \\
E \epsilon= & \omega_{e} \epsilon+\alpha a_{1},
\end{aligned}
$$

$(n=1,2, \ldots, N)$ where $\epsilon, a_{n}$ and $b_{n}$ are the excitation amplitudes in the atom and in the sublattices $\mathrm{A}$ and $\mathrm{B}$, respectively. A dark state corresponds to a localized state near the emitter, the degree of localization being measured by the participation ratio $P R=\left(\sum_{l}\left|c_{l}\right|^{2}\right)^{2} / \sum_{l}\left|c_{l}\right|^{4}$, where $\left\{c_{l}\right\}=\left\{a_{n}, b_{n}, \epsilon\right\}$ and $l=1,2, \ldots, 2 N+1$. For localized modes, $P R \sim 1$ while for extended states $P R \sim N$. Let us assume the atom in resonance with the electromagnetic modes of the cavities, i.e. $\omega_{e}=0$, and let us assume $N=2(2 s+1)$ with $s$ an arbitrary integer number. Numerical computation of the eigenvalues $E$ of the linear system equation (17) shows that for large $N$ the most localized mode, corresponding to the smallest value of the $\mathrm{PR}$, is the one with energy $E=0$, and reads explicitly

$$
a_{n}=0, \quad b_{n}=i^{n-1}, \quad, \epsilon=-\frac{2 \rho}{\alpha} .
$$

apart from a normalization factor. Note that this mode does not have any excitation in sublattice A, while the excitation is distributed in the emitter and uniformly in sublattice B. The PR of this mode is given by

$$
P R=\frac{\left[\left(\frac{2 \rho}{\alpha}\right)^{2}+N\right]^{2}}{\left(\frac{2 \rho}{\alpha}\right)^{4}+N} .
$$

Clearly, for large $N$ one has $P R \sim N$, i.e. this mode is not localized near the emitter and corresponds to a resonance state (the ratio between the amplitudes of excitation in the emitter and in the bath scales as $\sim \rho / \alpha$, diverging as $\alpha \rightarrow 0$, which is typical of a resonance state). This means that there is not any atom-field dark state in our system.

Solution of Eq. (8). For $t<T^{+}$we can neglect the sum over \pm in Eq. (8) of the main text, i.e.

$$
\dot{\varepsilon}(t)=-\frac{1}{2} \gamma_{0} \varepsilon(t)-\sum_{n=1}^{\infty} \gamma_{n}^{-} \varepsilon\left(t-n T^{-}\right) \Theta\left(t-n T^{-}\right),
$$

with $\epsilon(0)=1$. The above equation can be readily solved in Laplace domain. After introduction of the Laplace transform $\tilde{\epsilon}(s)=\int_{0}^{\infty} d t \epsilon(t) \exp (-s t)$, one obtains

$$
\tilde{\varepsilon}(s)=\left(s+\frac{\gamma_{0}}{2}-\frac{\gamma_{0}^{-}}{e^{s T^{-}}+1}\right)^{-1},
$$

where we assumed $N=2(2 l+1)$ with $l$ integer. Expanding in power of $x=e^{s T^{-}}$

$$
\tilde{\varepsilon}(s)=\frac{2}{2 s+\gamma_{0}}+\sum_{n=1}^{\infty} 4 \gamma_{0}^{-}(-1)^{n+1} e^{-n s T^{-}} \frac{\left(\gamma_{0}-2 \gamma_{0}^{-}+2 s\right)^{n-1}}{\left(\gamma_{0}+2 s\right)^{n+1}} .
$$

Returning into the time domain

$$
\varepsilon(t)=e^{-\frac{\gamma_{0} t}{2}}+\sum_{n=1}^{\infty}(-1)^{n+1} \Theta\left(t-n T^{-}\right) \gamma_{0}^{-}\left(t-n T^{-}\right)_{1} F_{1}\left(n+1,2,-\frac{\gamma_{0}\left(t-n T^{-}\right)}{2}\right),
$$

where ${ }_{1} F_{1}$ is the confluent hypergeometric function.

Decoherence dynamics for an odd number of sites in the ring. For the sake of completeness, let us discuss here the decay dynamics in a system with $N$ odd. As discussed in the main text, the dynamics of the coherences is accurately described by equation (8). In the case of an odd number of sites per sublattices, $\gamma_{n}^{ \pm}$can take imaginary values. As a consequence, and as shown in Fig. 4, the intermittent blockade displays a doubled period which can be understood considering the approximate linearized solution for $t<T^{+}$. If we call $\varepsilon_{R}(t)$ and $\varepsilon_{I}(t)$ respectively the real and the imaginary part of $\varepsilon(t)$, we have 

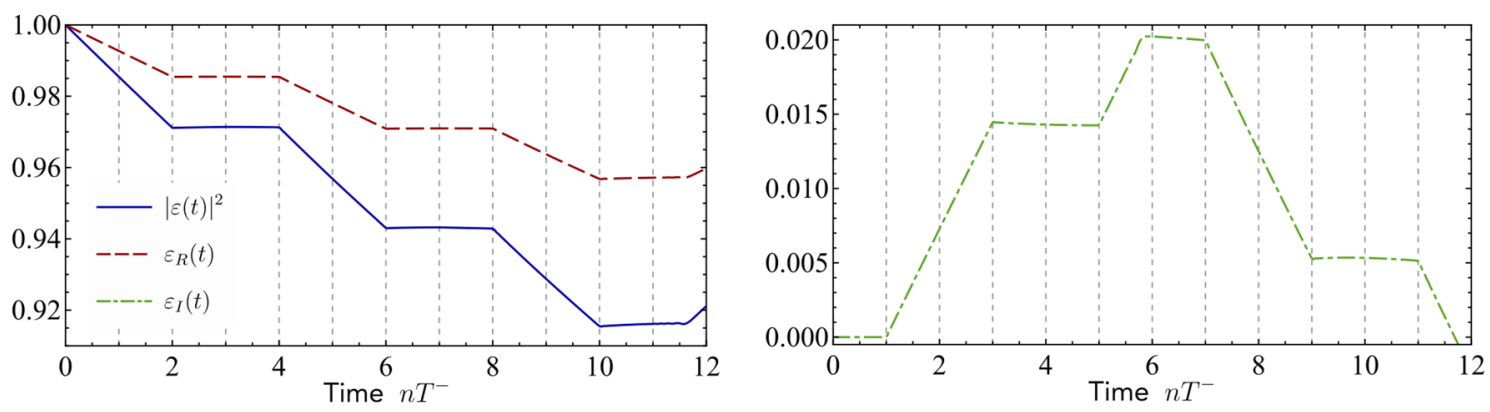

Figure 4. Evolution of the squared excitation amplitude $|\varepsilon(t)|^{2}$, solution of Eq. (6) in the main text and its real and imaginary parts $\varepsilon_{R}(t), \varepsilon_{I}(t)$, for $N=501, J=\rho=1$ and $\alpha=0.01$. In this regime intermittent blockade of the decay is observed until the time $t \sim 12 T^{-}$.

$$
\begin{gathered}
\varepsilon_{R}(t) \sim\left\{\begin{array}{llc}
1-\frac{1}{2} \gamma_{0}\left(t-2 m T^{-}\right) & \text {for } & (4 m) T^{-} \leq t \leq(4 m+1) T^{-} \\
1-\frac{1}{2} \gamma_{0}\left(t-2 m T^{-}\right) & \text {for } \quad(4 m+1) T^{-} \leq t \leq(4 m+2) T^{-} \\
1-\gamma_{0}(m+1) T^{-} & \text {for } \quad(4 m+2) T^{-} \leq t \leq(4 m+3) T^{-} \\
1-\gamma_{0}(m+1) T^{-} & \text {for } \quad(4 m+3) T^{-} \leq t \leq(4 m+4) T^{-}
\end{array}\right. \\
\varepsilon_{I}(t) \sim\left\{\begin{array}{llr} 
\pm \gamma_{0} m T^{-} & \text {for } \quad(4 m) T^{-} \leq t \leq(4 m+1) T^{-} \\
\pm \frac{1}{2} \gamma_{0}\left(t-(2 m+1) T^{-}\right) & \text {for } \quad(4 m+1) T^{-} \leq t \leq(4 m+2) T^{-} \\
\pm \frac{1}{2} \gamma_{0}\left(t-(2 m+1) T^{-}\right) & \text {for } \quad(4 m+2) T^{-} \leq t \leq(4 m+3) T^{-} \\
\pm \gamma_{0}(m+1) T^{-} & \text {for } \quad(4 m+3) T^{-} \leq t \leq(4 m+4) T^{-}
\end{array}\right.
\end{gathered}
$$

for $m=0,1,2,3, \ldots$, where for the imaginary part of the amplitude ' + ' corresponds to $N=4 s+1$ and '-' to $N=2(2 s+1)+1$, with $s$ integer. Thus, despite the short feedback period is still $T^{-}$, the dynamics displays four stages, in which the intermittency alternates between the real and imaginary parts of $\varepsilon(t)$ in periods of $2 T^{-}$. For the parameters considered in Fig. 4, this linearized solution is accurate until $t \sim 6 T^{-}$for the imaginary part and $t \sim 12 T^{+}$for the real part, in which the first and second slow wavepackets interact with the two level system. Because of $\gamma_{1}^{+}$is imaginary, the slow wavepacket needs two periods to affect the real part of the amplitude, and moreover, as in this early stage of the dynamics $\left|\varepsilon_{I}(t)\right| \ll\left|\varepsilon_{R}(t)\right|$ then $|\varepsilon(t)|^{2} \approx \varepsilon_{R}(t)^{2}$, and the intermittent blockade of the decay in $|\varepsilon(t)|^{2}$ is significant until $t \sim 12 T^{-}$in contrast to the case studied in the main text in which it ceases at $t \sim 6 T^{-}$.

Received: 24 July 2020; Accepted: 2 June 2021

Published online: 18 June 2021

\section{References}

1. Scully, M. O. \& Zubairy, M. S. Quantum Optics (Cambridge University Press, 1997).

2. Loudon, R. The Quantum Teory of Light 3rd edn (Oxford Univ. Press, 2000).

3. Haroche, S. \& Raimond, J. M. Exploring the Quantum: Atoms, Cavities, and Photons (Oxford University Press, 2006).

4. Weisskopf, V. \& Wigner, E. Berechnung der naturlichen Linienbreite auf Grund der Diracschen Lichttheorie. Zeitschrift für Physik 63, 54-73 (1930)

5. Goy, P., Raimond, J. M., Gross, M. \& Haroche, S. Observation of cavity-enhanced single-atom spontaneous emission. Phys. Rev. Lett. 50, 1903-1906 (1983).

6. Itano, W. M., Heinzen, D. J., Bollinger, J. J. \& Wineland, D. J. Quantum zeno effect. Phys. Rev. A 41, 2295 (1990).

7. Yablonovitch, E. Inhibited spontaneous emission in solid-state physics and electronics. Phys. Rev. Lett. 58, 2059-2062 (1987).

8. Misra, B. \& Sudarshan, E. C. G. The Zeno's paradox in quantum theory. J. Math. Phys. 18, 756 (1977).

9. Dicke, R. H. Coherence in spontaneous radiation processes. Phys. Rev. 93, 99 (1954).

10. Gross, M. \& Haroche, S. Superradiance: An essay on the theory of collective spontaneous emission. Phys. Rep. 93, 301 (1982).

11. Német, N., Carmele, A., Parkins, S. \& Knorr, A. Comparison between continuous- and discrete-mode coherent feedback for the Jaynes-Cummings model. Phys. Rev. A 100, 023805 (2019).

12. Shimizu, K. T., Woo, W. K., Fisher, B. R., Eisler, H. J. \& Bawendi, M. G. Surface-enhanced emission from single semiconductor nanocrystals. Phys. Rev. Lett. 89, 117401 (2002).

13. Anger, P., Bharadwaj, P. \& Novotny, L. Enhancement and quenching of single-molecule fluorescence. Phys. Rev. Lett. 96, 113002 (2006).

14. Noda, S., Fujita, M. \& Asano, T. Spontaneous-emission control by photonic crystals and nanocavities. Nat. Photon. 1, 449 (2007).

15. Ginzburg, P. et al. Spontaneous emission in non-local materials. Light: Sci. Appl. 6, e16273 (2017).

16. Petersen, J., Volz, J. \& Rauschenbeutel, A. Chiral nanophotonic waveguide interface based on spin-orbit interaction of light. Science 346, 67-71 (2014).

17. Ramos, T., Pichler, H., Daley, A. J. \& Zoller, P. Quantum spin dimers from chiral dissipation in cold-atom chains. Phys. Rev. Lett. 113, 237203 (2014).

18. Pichler, H., Ramos, T., Daley, A. J. \& Zoller, P. Quantum optics of chiral spin networks. Phys. Rev. A 91, 042116 (2015).

19. Metelmann, A. \& Clerk, A. A. Nonreciprocal photon transmission and amplification via reservoir engineering. Phys. Rev. X 5, 021025 (2015).

20. Lodahl, P. et al. Chiral quantum optics. Nature 541, 473 (2017).

21. Wang, P. et al. Synthesized magnetic field of a sawtooth superradiance lattice in Bose-Einstein condensates. npj Quantum Inf. 6 , $18(2020)$. 
22. Sanchez-Burillo, E., Wan, C., Zueco, D. \& González-Tudela, A. Chiral quantum optics in photonic sawtooth lattices. Phys. Rev. Res. 2, 023003 (2020).

23. Barik, S., Karasahin, A., Mittal, S., Waks, E. \& Hafezi, M. Chiral quantum optics using a topological resonator. Phys. Rev. B 101, $205303(2020)$.

24. Gustafsson, M. V. et al. Propagating phonons coupled to an artificial atom. Science 346, 207 (2014).

25. Guo, L., Grimsmo, A., Kockum, A. F., Pletyukhov, M. \& Johansson, G. Giant acoustic atom: A single quantum system with a deterministic time delay. Phys. Rev. A 95, 053821 (2017).

26. Kockum, A. F., Johansson, G. \& Nori, F. Decoherence-free interaction between giant atoms in waveguide quantum electrodynamics. Phys. Rev. Lett. 120, 140404 (2018).

27. Andersson, G., Suri, B., Guo, L., Aref, T. \& Delsing, P. Non-exponential decay of a giant artificial atom. Nat. Phys. 15, 1123 (2019).

28. González-Tudela, A., Sánchez Mũnoz, C. \& Cirac, J. I. Engineering and harnessing giant atoms in high-dimensional baths: A proposal for implementation with cold atoms. Phys. Rev. Lett. 122, 203603 (2019).

29. Guo, S. Wang, Y., Purdy, T. \& Taylor, J. Beyond Spontaneous Emission: Giant Atom Bounded in Continuum. Phys. Rev. A 102, $033706(2020)$

30. Guo, L., Frisk Kockum, A., Marquardt, F. \& Johansson, G., Oscillating bound states for a giant atom. Phys. Rev. Research 2, 043014 (2020)

31. Kannan, B. et al. Waveguide quantum electrodynamics with giant superconducting artificial atoms. Nature 583, 775-779 (2020)

32. Longhi, S. Photonic simulation of giant atom decay. Opt. Lett. 45, 3017-3020 (2020).

33. Kockum, A. F. Quantum optics with giant atoms - the first five years. In International Symposium on Mathematics, Quantum Theory, and Cryptography. Mathematics for Industry Vol. 33 (eds Takagi, T. et al.) https://doi.org/10.1007/978-981-15-5191-8 (Springer, 2020).

34. Grimsmo, A. L. Time-delayed quantum feedback control. Phys. Rev. Lett. 115, 060402 (2015).

35. Pichler, H. \& Zoller, P. Photonic circuits with time delays and quantum feedback. Phys. Rev. Lett. 116, 093601 (2016).

36. Whalen, S. J., Grimsmo, A. \& Carmichael, H. J. Open quantum systems with delayed coherent feedback. Quantum Sci. Technol. 2, 044008 (2017).

37. Cook, R. J. \& Milonni, P. W. Quantum theory of an atom near partially reflecting walls. Phys. Rev. A 35, 5081 (1987).

38. Dorner, U. \& Zoller, P. Laser-driven atoms in half-cavities. Phys. Rev. A 66, 023816 (2002).

39. Tufarelli, T., Ciccarello, F. \& Kim, M. S. Dynamics of spontaneous emission in a single-end photonic waveguide. Phys. Rev. A 87, 013820 (2013).

40. Tufarelli, T., Kim, M. S. \& Ciccarello, F. Non-Markovianity of a quantum emitter in front of a mirror. Phys. Rev. A 90, 012113 (2014).

41. Fang, Y.-L.L. \& Baranger, H. U. Waveguide QED: power spectra and correlations of two photons scattered off multiple distant qubits and a mirror. Phys. Rev. A 91, 053355 (2015).

42. Gu, X., Kockum, A. F., Miranowicz, A., Liu, Y. \& Nori, F. Microwave photonics with superconducting quantum circuits. Phys. Rep. 718-719, 1-102 (2017).

43. Fang, Y.-L.L., Ciccarello, F. \& Baranger, H. U. Non-Markovian dynamics of a qubit due to single-photon scattering in a waveguide. New J. Phys. 20, 043035 (2018).

44. Sinha, K. et al. Non-Markovian collective emission from macroscopically separated emitters. Phys. Rev. Lett. 124, 043603 (2020).

45. Carmele, A., Nemet, N., Canela, V. \& Parkins, S. Pronounced non-Markovian features in multiply excited, multiple emitter waveguide QED: Retardation induced anomalous population trapping. Phys. Rev. Res. 2, 013238 (2020).

46. Longhi, S. Superradiance paradox in waveguide lattices. Opt. Lett. 45, 3297-3300 (2020).

47. Ladd, T. et al. Quantum computers. Nature 464, 45-53 (2010).

48. Zanardi, P. \& Rasetti, M. Noiseless quantum codes. Phys. Rev. Lett. 79, 3306 (1997).

49. Lidar, D. A., Chuang, I. L. \& Whaley, K. B. Decoherence-free subspaces for quantum computation. Phys. Rev. Lett. 81, 2594 (1998).

50. Plenio, M. B. \& Huelga, S. F. Dephasing assisted transport: Quantum networks and bio-molecules. New J. Phys. 10, 113019 (2008).

51. Caruso, F., Chin, A. W., Datta, A., Huelga, S. F. \& Plenio, M. B. Highly efficient energy excitation transfer in light-harvesting complexes: The fundamental role of noise-assisted transport. J. Chem. Phys. 131, 105106 (2009).

52. Chin, A. W., Huelga, S. F. \& Plenio, M. B. Coherence and Decoherence in Biological System: Principles of Noise Assisted Transport and the origin of Long-lived Coherences. Phil. Trans. R. Soc. A 370, 3638 (2012).

53. Arnold, C., Vendrell, O., Welsch, R. \& Santra, R. Control of nuclear dynamics through conical intersections and electronic coherences. Phys. Rev. Lett. 120, 123001 (2018).

54. Gu, B. \& Franco, I. Generalized theory for the timescale of molecular electronic decoherence in the condensed phase. J. Phys. Chem. Lett. 9, 773 (2018).

55. Knight, P. L., Lauder, M. A. \& Dalton, B. J. Laser-induced continuum structure. Phys. Rep. 190, 1 (1990).

56. Zhu, S.-Y. \& Scully, M. O. Spectral line elimination and spontaneous emission cancellation via quantum interference. Phys. Rev. Lett. 76, 388 (1996).

57. Kiffner, M., Macovei, M., Evers, J. \& Keitel, C. H. Vacuum-induced processes in multilevel atoms. Prog. Opt. 55, 85 (2010).

58. Berman, P. R. Analysis of dynamical suppression of spontaneous emission. Phys. Rev. A 58, 4886 (1998).

59. Winkler, K. et al. Atom-molecule dark states in a Bose-Einstein condensate. Phys. Rev. Lett. 95, 063202 (2005).

60. Brandes, T. Coherent and collective quantum optical effects in mesoscopic systems. Phys. Rep. 408, 315 (2005).

61. Shapiro, M. \& Moshe, P. Quantum control of bound and continuum state dynamics. Phys. Rep. 425, 195 (2006).

62. Nakamura, H., Hatano, N., Garmon, S. \& Petrosky, T. Quasibound states in the continuum in a two channel quantum wire with an adatom. Phys. Rev. Lett. 99, 210404 (2007).

63. Longhi, S. Bound states in the continuum in a single-level Fano-Anderson model. Eur. Phys. J. B 57, 45 (2007).

64. Dreisow, F. et al. Adiabatic transfer of light via a continuum in optical waveguides. Opt. Lett. 34, 2405 (2009).

65. Schirmer, S. G. \& Wang, X. Stabilizing open quantum systems by Markovian reservoir engineering. Phys. Rev. A 81, 062306 (2010).

66. Filipp, S., van Loo, A. F., Baur, M., Steffen, L. \& Wallraff, A. Preparation of subradiant states using local qubit control in circuit QED. Phys. Rev. A 84, 061805(R) (2011).

67. Lombardo, F., Ciccarello, F. \& Palma, G. M. Photon localization versus population trapping in a coupled-cavity array. Phys. Rev. A 89, 053826 (2014).

68. Redchenko, E. S. \& Yudson, V. I. Decay of metastable excited states of two qubits in a waveguide. Phys. Rev. A 90, 063829 (2014).

69. Crespi, A. et al. Particle statistics affects quantum decay and fano interference. Phys. Rev. Lett. 114, 090201 (2015).

70. Hsu, C. W., Zhen, B., Stone, A. D., Joannopoulos, J. D. \& Soljacic, M. Bound states in the continuum. Nat. Rev. Mater. 1, 16048 (2016).

71. Facchi, P., Lonigro, D., Pascazio, S., Pepe, F. V. \& Pomarico, D. Bound states in the continuum for an array of quantum emitters. Phys. Rev. A 100, 023834 (2019).

72. Donarini, A. et al. Coherent population trapping by dark state formation in a carbon nanotube quantum dot. Nat. Commun. 10, 381 (2019).

73. Calajò, G., Fang, Y.-L.L., Baranger, H. U. \& Ciccarello, F. Exciting a bound state in the continuum through multiphoton scattering plus delayed quantum feedback. Phys. Rev. Lett. 122, 073601 (2019).

74. Mirhosseini, M. et al. Cavity quantum electrodynamics with atom-like mirrors. Nature 569, 692 (2019). 
75. Lidar, D. A. Review of decoherence free subspaces, noiseless subsystems, and dynamical decoupling. Adv. Chem. Phys. 154, 295 (2014).

76. Bocchieri, P. \& Loinger, A. Quantum recurrence theorem. Phys. Rev. 107, 337-338 (1957).

77. Roushan, P. et al. Chiral ground-state currents of interacting photons in a synthetic magnetic field. Nat. Phys. 13, 146-151 (2017).

78. Chen, Y. et al. Qubit architecture with high coherence and fast tunable coupling. Phys. Rev. Lett. 113, 220502 (2014).

\title{
Acknowledgements
}

We acknowledge the Spanish State Research Agency, through the Severo Ochoa and María de Maeztu Program for Centers and Units of Excellence in R\&D (MDM-2017-0711) and through the QUARESC project (PID2019-109094GB-C21 and -C22/ AEI / 10.13039/501100011033); we also acknowledge CSIC Research Platform PTI-001, the QUAREC project funded by CAIB, and funding from CAIB PhD program. GLG is funded by the Spanish Ministerio de Educación y Formación Profesional / Ministerio de Universidades and co-funded by the University of the Balearic Islands through the Beatriz Galindo program (BG20/00085). Sa. L. acknowledges support from MIUR through project PRIN Project 2017SRN-BRK QUSHIP and hospitality from IFISC under the "professors convidats" UIB program. Sa.L. thanks F. Ciccarello for useful discussions.

\section{Author contributions}

Sa. L., St. L. G. L. G. and R. Z. conceived the setup, Sa. L. and A. C. developed the analytical calculations, Sa.L. and G. L. G. performed the numerical simulations. All authors analysed the results and contributed to the writing of the manuscript.

\section{Competing interests}

The authors declare no competing interests.

\section{Additional information}

Correspondence and requests for materials should be addressed to G.L.G.

Reprints and permissions information is available at www.nature.com/reprints.

Publisher's note Springer Nature remains neutral with regard to jurisdictional claims in published maps and institutional affiliations.

\begin{abstract}
(c) (i) Open Access This article is licensed under a Creative Commons Attribution 4.0 International License, which permits use, sharing, adaptation, distribution and reproduction in any medium or format, as long as you give appropriate credit to the original author(s) and the source, provide a link to the Creative Commons licence, and indicate if changes were made. The images or other third party material in this article are included in the article's Creative Commons licence, unless indicated otherwise in a credit line to the material. If material is not included in the article's Creative Commons licence and your intended use is not permitted by statutory regulation or exceeds the permitted use, you will need to obtain permission directly from the copyright holder. To view a copy of this licence, visit http://creativecommons.org/licenses/by/4.0/.
\end{abstract}

(c) The Author(s) 2021 